\title{
Destruction of dimethyl ether and methyl formate by collisions with $\mathrm{He}^{+}$
}

\author{
Daniela Ascenzi ${ }^{1}$, Andrea Cernuto ${ }^{1}$, Nadia Balucani ${ }^{2,3,4}$, Paolo Tosi ${ }^{1}$, Cecilia Ceccarelli ${ }^{3,4}$, \\ Luca Matteo Martini ${ }^{1}$, and Fernando Pirani ${ }^{2}$ \\ 1 Department of Physics, University of Trento, Via Sommarive 14, 38123 Povo, Italy \\ e-mail: daniela.ascenzi@unitn.it \\ 2 Dipartimento di Chimica, Biologia e Biotecnologie, Università di Perugia, Via Elce di Sotto 8, 06123 Perugia, Italy \\ e-mail: nadia.balucani@unipg.it \\ 3 Univ. Grenoble Alpes, CNRS, IPAG, 38000 Grenoble, France \\ e-mail: cecilia.ceccarelli@univ-grenoble-alpes. fr \\ ${ }^{4}$ INAF-Osservatorio Astrofisico di Arcetri, Largo E. Fermi 5, 50125 Florence, Italy
}

Received 6 November 2018 / Accepted 11 March 2019

\begin{abstract}
Context. To correctly model the abundances of interstellar complex organic molecules (iCOMs) in different environments, both formation and destruction routes should be appropriately accounted for. While several scenarios have been explored for the formation of iCOMs via grain and gas-phase processes, much less work has been devoted to understanding the relevant destruction pathways, with special reference to (dissociative) charge exchange or proton transfer reactions with abundant atomic and molecular ions such as $\mathrm{He}^{+}, \mathrm{H}_{3}^{+}$and $\mathrm{HCO}^{+}$.

Aims. By using a combined experimental and theoretical methodology we provide new values for the rate coefficients and branching ratios (BRs) of the reactions of $\mathrm{He}^{+}$ions with two important iCOMs, namely dimethyl ether (DME) and methyl formate (MF). We also review the destruction routes of DME and MF by other two abundant ions, namely $\mathrm{H}_{3}{ }^{+}$and $\mathrm{HCO}^{+}$.

Methods. Based on our recent laboratory measurements of cross sections and BRs for the $\mathrm{DME} / \mathrm{MF}+\mathrm{He}^{+}$reactions over a wide collision energy, we extended our theoretical insights on the selectivity of the microscopic dynamics to calculate the rate coefficients $k(T)$ in the temperature range from 10 to $298 \mathrm{~K}$. We implemented these new and revised kinetic data in a general model of cold and warm gas, simulating environments where DME and MF have been detected.

Results. Due to stereodynamical effects present at low collision energies, the rate coefficients, BRs and temperature dependences here proposed differ substantially from those reported in KIDA and UDfA, two of the most widely used astrochemical databases. These revised rates impact the predicted abundances of DME and MF, with variations up to $40 \%$ in cold gases and physical conditions similar to those present in prestellar cores.

Conclusions. This work demonstrates that the accuracy of astrochemical models can be improved by a thorough characterisation of the destruction routes of iCOMs. The details of the chemical systems can, indeed, strongly affect their efficiency and significant deviations with respect to the commonly used Langevin model estimates are possible.
\end{abstract}

Key words. ISM: molecules - cosmic rays - ISM: abundances - molecular processes

\section{Introduction}

Interstellar complex organic molecules, hereafter called iCOMs, are C-bearing compounds containing at least six atoms (Herbst \& van Dishoeck 2009; Ceccarelli et al. 2017). Despite their relative simplicity with respect to what is considered a complex molecule in terrestrial terms, their detection in star forming regions (e.g. Rubin et al. 1971; Blake et al. 1987) has represented a challenge in our understanding of interstellar chemistry for decades. In particular, attention has so far focused on the possible formation routes, either via reactions occurring on the icy surfaces of the interstellar grains (e.g. Garrod \& Herbst 2006; Ruaud et al. 2015; Vasyunin et al. 2017) or in the gas phase (e.g. Vasyunin \& Herbst 2013; Balucani et al. 2015; Skouteris et al. 2017, 2018).

Much less effort has so far been spent on understanding the destruction pathways for these iCOMs, which also affect their abundances. Among all the possible destruction routes, there are three that are not only efficient but often the dominant ones for all species, namely the reactions with $\mathrm{He}^{+}$and the two most abundant molecular ions ${ }^{1}, \mathrm{H}_{3}{ }^{+}$and $\mathrm{HCO}^{+} . \mathrm{He}^{+}, \mathrm{H}^{+}$, and $\mathrm{H}_{2}{ }^{+}$ are created by the interaction of cosmic rays, which permeate the Galaxy, with $\mathrm{H}$ or $\mathrm{He}$ atoms, as well as $\mathrm{H}_{2}$ molecules. The $\mathrm{H}^{+}$and $\mathrm{H}_{2}{ }^{+}$ions formed in this way go on reacting with $\mathrm{H}_{2}$ to produce $\mathrm{H}_{3}{ }^{+}$. The latter then reacts with $\mathrm{CO}$ (the most abundant molecule after $\mathrm{H}_{2}$ in cold interstellar gas) and produces $\mathrm{HCO}^{+}$.

Although the ionisation cross sections of cosmic rays are very small (e.g. Padovani et al. 2009), they are the dominant source of gas ionisation in dense and UV-shielded environments. Likewise, despite their relatively low abundances $\left(\leq 10^{-6}\right.$ with respect to $\mathrm{H}_{2}$ ), $\mathrm{He}^{+}, \mathrm{H}_{3}{ }^{+}$and $\mathrm{HCO}^{+}$dominate the destruction of the vast majority of iCOMs. In general, the reactions of iCOMs with $\mathrm{H}_{3}{ }^{+}$and $\mathrm{HCO}^{+}$result in a proton transfer which can be, but is not necessarily, dissociative. Protonated species, in turn, can undergo dissociative electron recombination, thereby producing neutral molecules. In this last process, besides losing a $\mathrm{H}$

1 In water-rich regions, the $\mathrm{H}_{3} \mathrm{O}^{+}$ion can also play a dominant role. 
atom, the molecular species can undergo severe fragmentation (e.g. Geppert et al. 2006). Conversely, $\mathrm{He}^{+}$or $\mathrm{H}^{+}$react mostly by charge exchange. Because of the large energy involved in the case of iCOMs, charge exchange is often dissociative, with fragmentation likely more pronounced with $\mathrm{He}^{+}$due to the larger exotermicity. These processes are believed to be highly efficient and are included in all astrochemical models.

Despite the importance of these destruction pathways of iCOMs, studies devoted to characterising the reaction rates are scarce. Of the nearly one thousand reactions listed in the databases used in astrochemical models, KInetic Database for Astrochemistry ${ }^{2}$ (KIDA: Wakelam et al. 2012) and UMIST Database for Astrochemistry ${ }^{3}$ (UDfA: McElroy et al. 2013), with a rapid survey we find that a rather small number of them have been studied in detail, either in laboratories or theoretically: no more than $\sim 10 \%$. For the vast majority of the reactions with $\mathrm{He}^{+}, \mathrm{H}_{3}{ }^{+}$, and $\mathrm{HCO}^{+}$both the rate coefficients and product branching ratios (BRs) are estimated based on common sense and analogous reactions. Very likely many of them are roughly correct, but it is difficult, if not impossible, to say so a priori.

With this work, we aim to start filling this crucial gap in our knowledge of astrochemistry. Specifically, we report an experimental and theoretical study of the reaction of $\mathrm{He}^{+}$with two important iCOMs: dimethyl ether $\left(\mathrm{CH}_{3} \mathrm{OCH}_{3}\right.$ : hereinafter DME) and methyl formate $\left(\mathrm{HCOOCH}_{3}\right.$ : hereinafter $\left.\mathrm{MF}\right)$. We chose these two species for the following reasons:

1. They are detected in hot cores, corinos and even cold prestellar cores with abundances, with respect to $\mathrm{H}_{2}$, that range from $\sim 10^{-10}$ to $\sim 10^{-7}$ (Jaber et al. 2014; Jimenez-Serra et al. 2016).

2. Two competing theories postulate that DME and MF are formed either on the grain surfaces (e.g. Garrod \& Herbst 2006; Shingledecker et al. 2018) or in the gas phase (Balucani et al. 2015; Skouteris et al. 2019), so that their study can give us a guidance as to which theory is more correct.

3. No measurements or theoretical studies exist in the literature about the DME and MF destruction by $\mathrm{He}^{+}$.

4. Previous works have provided information on the rate coefficients and product BRs of the reactions of DME and MF with $\mathrm{H}_{3}{ }^{+}$and $\mathrm{HCO}^{+}$(e.g. Tanner et al. 1979; Lee et al. 1992; Lawson et al. 2012), as well as on the dissociative recombination with electrons of methoxymethyl and protonated DME cations (e.g. Hamberg et al. 2010), so that a comparison is possible.

5. They are targets of the large observational programme IRAM-NOEMA SOLIS ${ }^{4}$ (Ceccarelli et al. 2017), so that their abundances are and will be measured in several different environments, which will allow us to test the various formation theories (point 1), if, and only if, we can be sure of the destruction routes.

The present article has been stimulated by recent measurements performed by some of the present authors under high resolution conditions of the absolute total cross-section for the reaction of $\mathrm{He}^{+}$with $\mathrm{DME}$ and $\mathrm{MF}$ in a wide range of collision energies (Cernuto et al. 2017, 2018). The results of this study are summarised in Fig. 1 and briefly described in Sect. 3.1. Detailed analysis of these results suggests that the dynamical evolution of such systems is driven at a microscopic level, by a strong anisotropy in the interaction potential energy hypersurfaces, which presumably also affects their macroscopic behaviour.

\footnotetext{
http://kida.obs.u-bordeaux1.fr

http://udfa.ajmarkwick.net/

4 https://solis.osug.fr/
}

In this article, we first review the rate coefficient and BRs values for the $\mathrm{DME}+\mathrm{He}^{+}$and $\mathrm{MF}+\mathrm{He}^{+}$reactions that are available in the astrochemical databases KIDA and UDfA (Sect. 2). We then provide new rate coefficients and BRs, based on our recent laboratory measurements and theoretical insights on the selectivity of the microscopic dynamics (Sect. 3). For completeness, we also review the reactions of DME and MF with the other molecular ions abundant in the interstellar gas, namely $\mathrm{HCO}^{+}$ and $\mathrm{H}_{3}{ }^{+}$(Sect. 4). Finally, we discuss the implications for astrochemical model predictions (Sect. 5) and the major conclusions of this study (Sect. 6).

\section{Brief review of the DME and MF reactions with $\mathrm{He}^{+}$in the astrochemical databases}

\subsection{Methodology}

In the KIDA and UDfA databases, the suggested values for rate constants as a function of temperature are based on a modified Arrhenius equation, using the parameters from the OSU2009 gas-phase chemistry database of Herbst (Herbst 2009).

$k(T)=\alpha\left(\frac{T}{300}\right)^{\beta} e^{-\gamma / T}\left[\mathrm{~cm}^{3} \mathrm{~s}^{-1}\right]$.

For ion-neutral reactions, $\alpha$ is a parameter specific for each reaction, while the other two parameters are usually assumed to be $\beta=-0.50, \gamma=0.00$ and the temperature $T$ is in the range from $10 \mathrm{~K}$ to $280 \mathrm{~K}$ (in the KIDA database) or from $10 \mathrm{~K}$ to $41000 \mathrm{~K}$ (in the UDfA database).

It should be noted that the above expression is an approximation (valid for low temperatures) of a more general expression deriving from a classical dynamic treatment of capture in a non central potential. Such a capture model was developed by $\mathrm{Su}$ and Chesnavich (Chesnavich et al. 1980; Su \& Chesnavich 1982) and is a combined variational transition state theory and classical trajectory study of thermal energy collisions between an ion (treated as a point charge particle) and a polar molecule (treated as a two-dimensional rigid rotor). In the model, the interaction potential between the ion and the polar molecules includes only the ion-induced dipole and ion-permanent dipole terms.

Thermal rate coefficients determined from the calculations have been fitted to analytical expressions given in terms of the parameter $x$ defined as

$x=\mu_{\mathrm{D}} /\left(2 \alpha_{\mathrm{e}} k_{\mathrm{B}} T\right)^{0.5}$

where $\mu_{\mathrm{D}}$ is the dipole moment of the neutral, $\alpha_{\mathrm{e}}$ is the average electronic polarisability of the neutral and $k_{\mathrm{B}}$ is the Boltzmann constant.

For $x \geq 2$, the Su \& Chesnavich formula for the rate constant $k(T)$ can be written as follows

$k(T)=c_{1}+c_{2} T^{-0.5}$

with $c_{1}=0.62 k_{\mathrm{L}}$ and $c_{2}=2.1179\left(\mu_{\mathrm{D}} q\right) /\left(\mu k_{\mathrm{B}}\right)^{-0.5}$ and where $k_{\mathrm{L}}$ is the Langevin rate constant:

$k_{\mathrm{L}}=2 \pi q\left(\alpha_{\mathrm{e}} / \mu\right)^{0.5}$

with $\mu$ being the reduced mass of the reactants and $q$ the electric charge of the ion. In expression (3), when cgs-esu units are utilised, the $k(T)$ values are obtained in $\mathrm{cm}^{3} \mathrm{~s}^{-1}$. Additionally, when $c_{1} \ll c_{2} T^{-0.50}$, which is more likely at low temperatures, Eq. (3) reduces to (1). For $x<2$ a more complex 


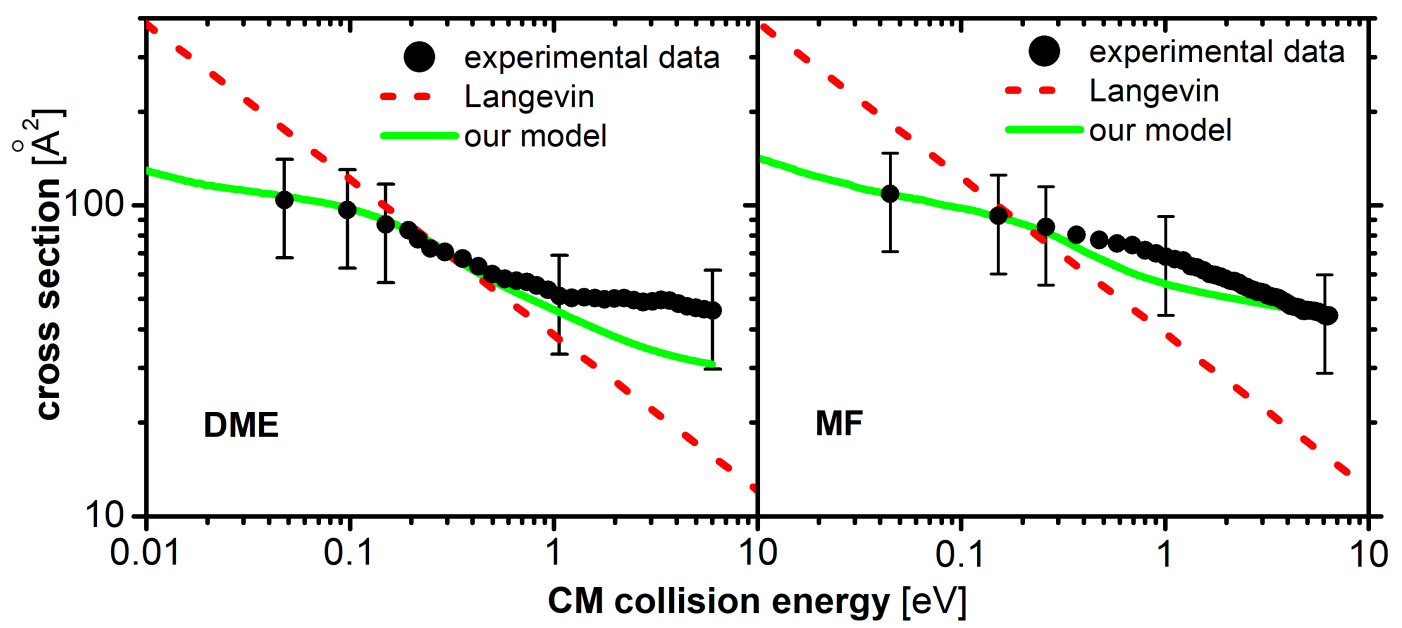

Fig. 1. Experimental and theoretical cross-sections for the charge-exchange reaction of $\mathrm{He}^{+}-\mathrm{DME}\left(\right.$ left panel) and $\mathrm{He}^{+}-\mathrm{MF}($ right panel), as a function of the collision energy. The black points represent the measured total cross-sections (Cernuto et al. 2017, 2018). Error bars represent a $35 \%$ error on the absolute values of the cross-section (relative cross-sections have smaller uncertainties). The curves represent the total crosssections calculated by our model, which is based on an improved Landau-Zener-Stückelberg approach (green lines), and a simple Langevin model (red dashed lines), (see text for detail).

expression than Eq. (3) is obtained (see Su \& Chesnavich 1982 and Wakelam et al. 2010 for the detailed expressions).

The Su \& Chesnavich model has been reported to work well for neutral species with linear, symmetric tops or asymmetric top geometries. However, being a classical treatment, it is valid only at temperatures above which the rotational motion can be considered classical, in other words, when $k_{\mathrm{B}} T$ is much higher than the relevant rotational constants of the neutral molecule. For most species, the classical treatment is still reliable down to $10-20 \mathrm{~K}$, while at lower temperatures $(\sim 0.1-10 \mathrm{~K})$ a semiclassical regime applies in which quantum effects in the hinderedrotor type motion should be considered, while the relative translational motion can still be treated classically. Using the statistical adiabatic channel model developed by J. Troe and collaborators (Troe 1996), the capture of rotationally state-selected and unselected polar molecules by ions in such low temperature regimes has been studied in details (Maergoiz et al. 2009 and references therein). The noticeable result is that an expression similar to Eq. (3) can be used where $\mu_{\mathrm{D}}$ is substituted by an effective dipole moment of the neutral reactant $\left(\mu_{\mathrm{D}, \mathrm{eff}}\right)$, which must be calculated following the lines indicated in Maergoiz et al. (2009). However, the differences might be so small (in the case of $\mathrm{H}_{2} \mathrm{O}$ as neutral partner $\mu_{\mathrm{D} \text {,eff }} \approx 1.07 \mu_{\mathrm{D}}$ ) that they lie within the uncertainty of the fits and calculations using the Su \& Chesnavich model.

\subsection{The case of dimethyl ether}

In the KIDA and UDfA databases, the reaction of $\mathrm{He}^{+}$ions with DME is reported to give the following two products, each with a BR of $50.0 \%$ :

$$
\begin{aligned}
& \mathrm{CH}_{3} \mathrm{OCH}_{3}+\mathrm{He}^{+} \rightarrow \mathrm{H}+\mathrm{He}+\mathrm{H}_{2} \mathrm{CO}+\mathrm{CH}_{3}^{+} \\
& \mathrm{CH}_{3} \mathrm{OCH}_{3}+\mathrm{He}^{+} \rightarrow \mathrm{He}+\mathrm{CH}_{3}+\mathrm{H}_{2} \mathrm{COH}^{+} .
\end{aligned}
$$

The suggested values for the total rate constant as a function of temperature are given in terms of the modified Arrhenius Eq. (1), with the following parameters: $\alpha=2.64 \times 10^{-9}$ in KIDA and $\alpha=2.00 \times 10^{-9}$ in UDfA, with $\beta=-0.50, \gamma=0.00$ in both. Also in this case, the $k(T)$ expression reported in the KIDA and UDfA databases is an approximation (valid for low temperatures) of the more general Eq. (3).

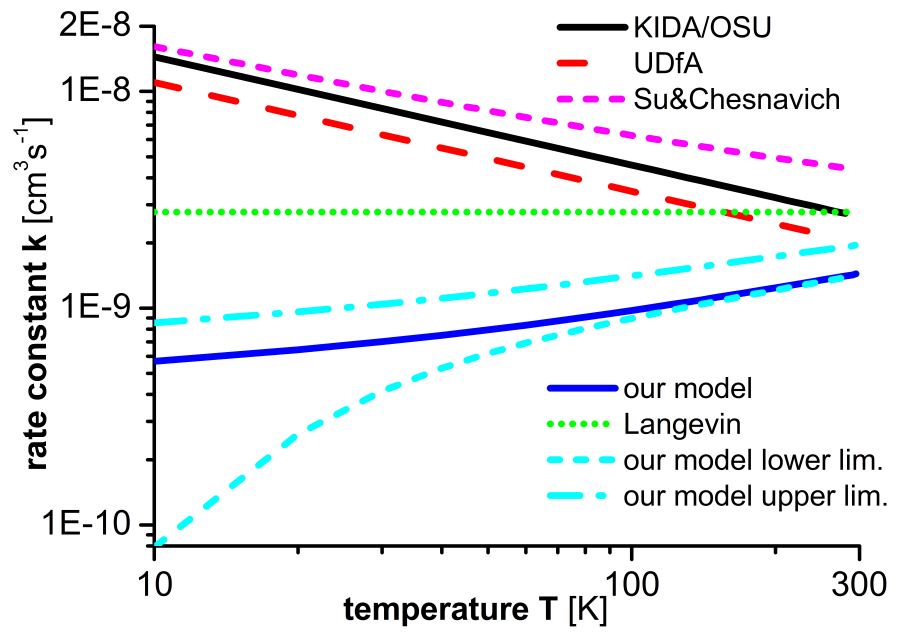

Fig. 2. Temperature dependence of the total rate constant $k$ for the reaction of $\mathrm{He}^{+}$with DME. Solid black line: recommended values from KIDA database according to Eq. (1); dashed red line: recommended values from UDfA according to Eq. (1); dotted green line: Langevin rate constant $k_{\mathrm{L}}$ according to Eq. (4); dashed pink line: classical trajectory scaling according to Eq. (3) (Su \& Chesnavich 1982); solid blue line: our calculations; cyan dotted-dashed line: our calculations, upper limit estimate; cyan dashed line: our calculations, lower limit estimate (see text).

In the DME case $\left(\mu_{\mathrm{D}}=1.3 \mathrm{D}, \alpha_{\mathrm{e}}=5.16 \AA^{3}, \mu=6.112 \times\right.$ $10^{-27} \mathrm{~kg}$ ), the Langevin rate constant is $k_{\mathrm{L}}=2.77 \times 10^{-9} \mathrm{~cm}^{3} \mathrm{~s}^{-1}$ and the use of expression (1) rather than (3) at $T=10 \mathrm{~K}$ leads to an underestimation of the rate constant by about $10 \%$, a value which rises to about $39 \%$ at $T=280 \mathrm{~K}$. The rate constants as a function of temperature over the $10-300 \mathrm{~K}$ range are calculated according to the recommendation of KIDA and UDfA databases, as well as using the trajectory scaling method of Eq. (3), and the values are reported in Fig. 2.

Finally, the KIDA database gives $k=7.23 \times 10^{-9} \mathrm{~cm}^{3} \mathrm{~s}^{-1}$ for both reactions (5) and (6) at $10 \mathrm{~K}$, with a total $k$ for the title reaction equal to $1.45 \times 10^{-8} \mathrm{~cm}^{3} \mathrm{~s}^{-1}$. At $280 \mathrm{~K}$ (upper limit of Eq. (1)), the model gives a total $k$ of $2.74 \times 10^{-9} \mathrm{~cm}^{3} \mathrm{~s}^{-1}$. In the 
UDfA database, the $k_{300 \mathrm{~K}}$ is fixed to $2.00 \times 10^{-9} \mathrm{~cm}^{3} \mathrm{~s}^{-1}$, giving a rate constant at $10 \mathrm{~K}$ equal to $1.10 \times 10^{-8} \mathrm{~cm}^{3} \mathrm{~s}^{-1}$ and $2.07 \times$ $10^{-9} \mathrm{~cm}^{3} \mathrm{~s}^{-1}$ at $280 \mathrm{~K}$.

\subsection{The case of methyl formate}

In the KIDA and UDfA databases, the reaction of $\mathrm{He}^{+}$ions with MF is reported to give only the following channel (hence with a BR of $100.00 \%$ ):

$\mathrm{HCOOCH}_{3}+\mathrm{He}^{+} \rightarrow \mathrm{He}+\mathrm{CH}_{3}+\mathrm{HOCO}^{+}$.

The suggested values for the rate constant as a function of temperature are given in terms of the modified Arrhenius Eq. (1), with the following parameters: $\alpha=3.54 \times 10^{-9}$ in KIDA and $\alpha=3.00 \times 10^{-9}$ in UDfA, with $\beta=-0.50, \gamma=0.00$ in both. Also in this case, the $k(T)$ expression reported in the KIDA and UDfA databases is an approximation (valid for low temperatures) of the more general Eq. (3).

For MF, $\left(\mu_{\mathrm{D}}=1.77 \mathrm{D}, \alpha_{\mathrm{e}}=5.3 \AA^{3}, \mu=6.227 \times 10^{-27} \mathrm{~kg}\right)$ the Langevin rate constant is $k_{\mathrm{L}}=2.78 \times 10^{-9} \mathrm{~cm}^{3} \mathrm{~s}^{-1}$ and the use of expression (1) rather than (3) at $T=10 \mathrm{~K}$ leads to an underestimation of the rate constant by about $8 \%$, a value which rises to about $32 \%$ at $T=280 \mathrm{~K}$.

Rate constant values as a function of temperature in the range $10-300 \mathrm{~K}$ were calculated using the expressions reported in the KIDA and UDfA databases, as well as with the trajectory scaling method of Eq. (3) and results are shown in Fig. 3. Finally, the KIDA and UDfA values are equal to $1.94 \times 10^{-8} \mathrm{~cm}^{3} \mathrm{~s}^{-1}$ and $1.64 \times 10^{-8} \mathrm{~cm}^{3} \mathrm{~s}^{-1}$, respectively, at $10 \mathrm{~K}$, and $3.66 \times 10^{-9} \mathrm{~cm}^{3} \mathrm{~s}^{-1}$ and $3.11 \times 10^{-9} \mathrm{~cm}^{3} \mathrm{~s}^{-1}$ at $280 \mathrm{~K}$.

\section{New reaction rate coefficients and branching ratios for $\mathrm{He}^{+} / \mathrm{DME}$ and $\mathrm{He}^{+} / \mathrm{MF}$}

\subsection{Our model of the total cross-sections}

As mentioned in the Introduction, absolute total cross-sections as a function of the collision energy in the $0.04-6 \mathrm{eV}$ energy range for collisions of $\mathrm{He}^{+}$ions with DME and MF were recently measured using a guided ion beam mass spectrometer (Cernuto et al. 2017, 2018; see Fig. 1). In order both to analyse the results and properly rationalise all our experimental findings, this work focuses on two main aspects.

First, the entrance and exit channels of the potential energy surfaces (PESs) of the two reactions were characterised. The non-covalent nature of the interactions implies that each PES depends on the balance between repulsion and the combined dispersion and induction attraction. For the entrance channels, the electrostatic contribution due to the long range ion-permanent dipole interaction components has also been added. We find that the interaction anisotropy becomes comparable to or larger than the mean rotational energy of DME and MF at room temperature, already at large separation distance from $\mathrm{He}^{+}$(i.e. 15-20 $\AA$ ). Therefore, the approach of $\mathrm{He}^{+}$during the collision leads each molecule to probe an increasing electric field gradient that becomes sufficient to transform the molecular free rotation into hindered rotation and, afterwards, to drive the system in a pendular state. The latter is a particular case of bending motion confined in narrow angular cones around the most stable configuration of the ion-molecule collision complex. Such "natural" molecular polarisation phenomena become important in systems driven by strongly anisotropic long-range forces, as is the case for the present reactions. These effects are expected to grow in significance at low collision energies, causing changes in reactivity due

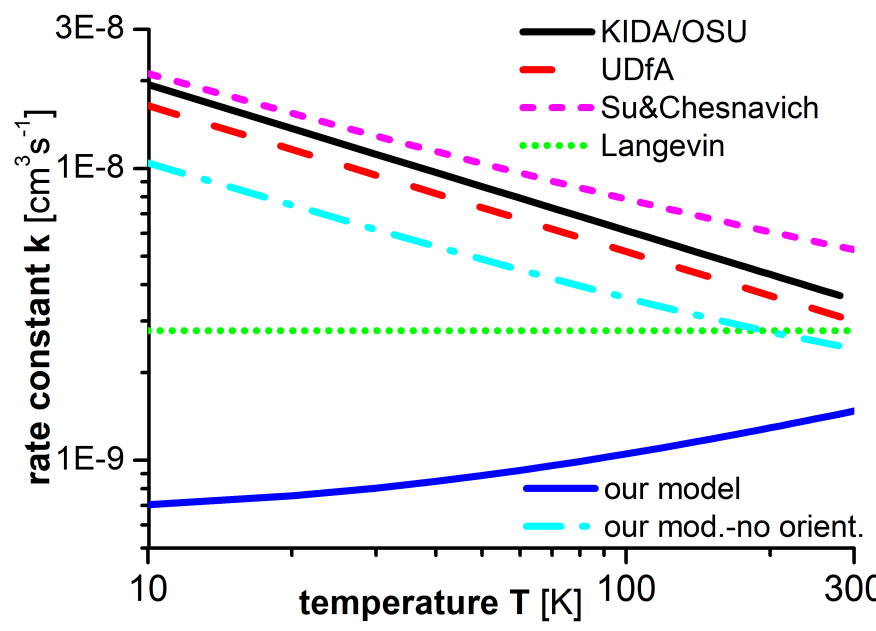

Fig. 3. Temperature dependence of the total rate constant $k$ for the reaction of $\mathrm{He}^{+}$with MF. The curve colours are as in Fig. 2, except for the dashed-dotted cyan line which corresponds to the data calculated using our model and assuming no preferential orientation of the colliding system.

to changes in the structure and stability of the collision complexes. They can therefore be regarded as "stereodynamical effects".

Secondly, we used a semi-classical model to evaluate the transition probability from the entrance to the exit channels. The model was developed ad hoc to give a unified description of the collision dynamics of both systems and to interpret the experimental results shown in Fig. 1. In the model, the transfer of an electron from DME/MF to $\mathrm{He}^{+}$is assumed to proceed via non-adiabatic transitions located at the crossings between the entrance $\left(\mathrm{He}^{+}-\mathrm{DME} / \mathrm{MF}\right)$ and exit $\left(\mathrm{He}-\mathrm{DME}^{\bullet+} / \mathrm{MF}^{\bullet+}\right)$ sections of the PESs given in the diabatic representation. Positions and energies of the crossings indicate that $\mathrm{He}^{+}$captures an electron exclusively from an inner molecular orbital of DME/MF, thus generating a highly excited/unstable molecular ion that can readily dissociate.

The probability of the non-adiabatic event at each crossing has been evaluated using a generalised Landau-ZenerStückelberg approach, taking into account the symmetry of the electron density distribution of the molecular orbitals of DME and MF from which the electron is removed. To this end, the non-adiabatic coupling has been represented, according to previous treatments (Gislason \& Sachs 1975; Gislason et al. 2007; Falcinelli et al. 2016), as a constant value modulated by an angular term. The latter brings the coupling to zero for geometries with null overlap between the atomic $s$ orbital of $\mathrm{He}^{+}$and the relevant molecular orbitals, while it makes the coupling maximum for geometries giving the best overlap (corresponding to the maximum electron density for the relevant molecular orbitals). In some cases, it was necessary to assume that, in absence of an appreciable overlap between the molecular and atomic orbitals exchanging the electron, the non-adiabatic events are exclusively due to the Coriolis (rotation-orbit) coupling. As demonstrated by the data plotted in Fig. 1, our approach is able to reproduce the experimental cross sections (especially at low collision energies), while a simple Langevin model fails.

\subsection{Rate coefficients: computational method}

The rate coefficients as a function of temperature, $k(T)$, have been obtained by averaging the total cross-sections calculated 
using our model (briefly described in Sect. 3.1 and shown in Fig. 1) over a Maxwell-Boltzmann distribution of collision energies, $E$, between $E_{\min }$ and $E_{\max }$, as follows:

$k(T)=\left(\frac{1}{\pi \mu}\right)^{1 / 2} \cdot\left(\frac{2}{k_{\mathrm{B}} T}\right)^{3 / 2} \cdot \int_{E_{\min }}^{E_{\max }} \sigma(E) \cdot E \cdot e^{-E / k_{\mathrm{B}} T} \mathrm{~d} E$.

This treatment, based on a generalised Landau-ZenerStückelberg approach, defines the energy range within which the cross-sections calculated using a semi-classical method are valid. Specifically, the adopted semi-classical methodology is applicable when the de Broglie wavelength $\left(\lambda_{\mathrm{DB}}\right)$ for the relative motion of the colliding partners is smaller than the distances at which the potential wells in the entrance and exit channels are located. For example, for DME these distances range between $1.5 \AA$ to $3 \AA$, while for MF they range between $2 \AA$ to $4 \AA$. Crossings among the entrance and exit potential energy curves are located within the same range. To obtain a conservative estimate, we have assumed that $\lambda_{\mathrm{DB}}=1.5 \AA$, resulting in a minimum energy $E$ of about $10 \mathrm{meV}$, below which the semi-classical treatment is not fully reliable. We note that, to obtain an accurate estimate of $k(T)$ at low temperatures (without incurring errors from numerical artefacts), cross-section values at energies smaller than $10 \mathrm{meV}$ have to be included in the integral of Eq. (8).

\subsection{Dimethyl ether}

In order to evaluate the most appropriate $E_{\min }$ to use in the integral of Eq. (8), we compared the result of Eq. (8) when $\sigma(E)$ is taken to be equal to the Langevin cross-section $\sigma_{\text {Langevin }}(E)=$ $q \sqrt{\frac{\pi \alpha}{2 \varepsilon_{0} E}}$ (shown in Fig. 1) with the rate coefficient given by the Langevin model (Eq. (4)).

We carried out these computations for the case of DME, for which the Langevin rate coefficient is $2.77 \times 10^{-9} \mathrm{~cm}^{3} \mathrm{~s}^{-1}$, and it is independent of $T$. We fixed the upper energy limit $E_{\max }$ at $10000 \mathrm{meV}$ and varied $E_{\min }$ to obtain an estimate of the error. At $300 \mathrm{~K}$, the numerical error on the calculated rate coefficient is negligible already when $E_{\min }$ is set equal to $0.1 \mathrm{meV}$. However, at $10 \mathrm{~K}$, it is necessary to decrease $E_{\min }$ to $0.01 \mathrm{meV}$ to have a relative error within $0.1 \%$.

Therefore, we set $E_{\max }$ to $10000 \mathrm{meV}$ and $E_{\min }$ to $0.01 \mathrm{meV}$ in order to compute the rate coefficients with the cross-sections from our model (Cernuto et al. 2017, 2018: see Sect. 3.1). However, since our cross-sections would strictly apply only up to energies larger than about $10-30 \mathrm{meV}$, we also calculated the rate coefficients over the 0.01 to $10 \mathrm{meV}$ range using two different models for the cross-section over this range. These models are described below:

1. Cross-sections increasing with increasing energy $E$ : a function $\propto E^{2}$ has been chosen to represent $\sigma$;

2. Cross-sections decreasing with increasing energy $E$ : in this case, the Landau-Zener-Stückelberg treatment has been used, but the strength of the Coriolis coupling has been modulated by obtaining calculated cross-sections at the upper limit of the experimental determinations.

Using the cross-sections from the first case $\left(\sigma \propto E^{2}\right)$, the resulting rate coefficient at $10 \mathrm{~K}$ is lower by a factor of 7.7 with respect to the one calculated with our model, whereas at $300 \mathrm{~K}$ the two values coincide. Using the cross-sections from the second case, the resulting rate coefficient is higher by a factor $1.4-1.5$ over the whole range of temperature. The resulting curves are shown in Fig. 2: the first case likely represents a lower limit while the second case is likely an upper limit to the rate coefficients, as labelled in the figure. Similar values for upper and lower limits to $k$ are expected to be valid also for the MF case (see Sect. 3.4).

\subsection{Methyl formate}

In the MF case, in addition to computing the rate coefficients using our model for the cross-sections (Sect. 3.1), we have also performed a simulation without the assumption of a strong stereochemical effect in the reaction induced by the formation of pendular states (i.e. preferred orientations of the polar molecule in the electric field generated by $\mathrm{He}^{+}$), and neglecting the Coriolis coupling. Should this be the case, the dynamical calculations must include contributions to the cross-sections from all the possible approach geometries of $\mathrm{He}^{+}$and MF. Therefore, contributions from points over the whole sphere around the neutral species must be considered rather than just selected contributions from a limited number of configurations within narrow angular cones around the most attractive geometries, such as is the case for our model. For a proper mapping of all the possible approach geometries, a total of 74 equally spaced points on the sphere around the centre of mass of the reacting system have been considered.

The resulting rate coefficients obtained with the crosssections calculated independent of orientation are shown in Fig. 3, with the label "our mod.-no orient". The rate coefficient at $10 \mathrm{~K}$ is more than one order of magnitude larger than the one computed using our model that considers the orientation dependence of reactivity. The difference between the two estimates diminishes with increasing temperature but it is still about $30 \%$ at $300 \mathrm{~K}$.

We emphasise, however, that the cross-sections calculated assuming an important orientation effect among the reactants and the formation of pendular states, that is, the model of Sect. 3.1, are in good agreement with the experimental results (Fig. 1). Conversely, calculations in the absence of stereochemical effect are not able to reproduce the experimental results. This provides a strong evidence that stereo-chemical effects play a role in the collisions of $\mathrm{He}^{+}$with polar neutrals and should not be disregarded when seeking to obtain reasonable estimates of rate constants. In the following, therefore, we will use the rate coefficients obtained using our model from Sect. 3.1.

\subsection{Comparison with previous rate coefficients}

Our new rate coefficients are compared with recommended values from KIDA, UDfA and classical trajectory scaling for the $\mathrm{He}^{+}$-DME case in Fig. 2, while the corresponding values for the $\mathrm{He}^{+}-\mathrm{MF}$ case are presented in Fig. 3. The differences between the calculated rate coefficients and the estimates of KIDA and UDfA databases are evident.

For DME, we obtain values of $k=5.69 \times 10^{-10} \mathrm{~cm}^{3} \mathrm{~s}^{-1}$ at $10 \mathrm{~K}$ and $1.44 \times 10^{-9} \mathrm{~cm}^{3} \mathrm{~s}^{-1}$ at $300 \mathrm{~K}$. At $10 \mathrm{~K}$, the KIDA database overestimates the rate coefficient by a factor $\sim 26$, while the UDfA value is $\sim 20$ times higher than our rate. At $300 \mathrm{~K}$, the differences between the calculated and estimated rate constants decrease, with the KIDA value being $\sim 2$ times higher than our value and the UDfA one being only $\sim 1.5$ higher. For $\mathrm{MF}$, we obtain values of $k=7.05 \times 10^{-10} \mathrm{~cm}^{3} \mathrm{~s}^{-1}$ at $10 \mathrm{~K}$ and $1.48 \times 10^{-9} \mathrm{~cm}^{3} \mathrm{~s}^{-1}$ at $300 \mathrm{~K}$. At $10 \mathrm{~K}$, KIDA and UDfA recommend values are $\sim 27$ and $\sim 23$ times higher, respectively, than our value. Around $300 \mathrm{~K}$, KIDA and UDfA overestimate the rate constants by factors of $\sim 2.5$ and $\sim 2$, respectively. 
Table 1. Product branching ratios (BRs) for the reaction $\mathrm{He}^{+}+\mathrm{DME}$.

\begin{tabular}{lccccc}
\hline \hline Product & $m / z$ & \multicolumn{3}{c}{ BRs (\%) } & \\
\cline { 3 - 4 } \cline { 3 - 4 } & & Exp. values & KIDA & & UDfA \\
\hline $\mathrm{CH}_{2}{ }^{+}$ & 14 & $7.3 \pm 0.8$ & 0 & \\
$\mathrm{CH}_{3}{ }^{+}$ & 15 & $38.5 \pm 3.7$ & 50 & & 100 \\
$\mathrm{HCO}^{+}$ & 29 & $53.6 \pm 5.3$ & 0 & & 0 \\
$\mathrm{OCH}_{3}{ }^{+}$ & 31 & $0.4 \pm 0.7$ & 50 & & 0 \\
\hline
\end{tabular}

Notes. Values are obtained, at a collision energy in the CM frame of $\sim 1.6 \mathrm{eV}$, by averaging results measured at pressures of DME between $2 \times 10^{-7}$ and $5 \times 10^{-6}$ mbar. BRs show no change with varying collision energy over the explored range.

In summary, our results show the database values to be significant overestimates, especially at $10 \mathrm{~K}$. It is worth noting that the rate coefficients of the astrochemical databases exhibit a negative temperature dependence (anti-Arrhenius behaviour), while those calculated by us show a positive Arrhenius dependence, even though the present charge transfer processes are both exothermic and barrierless. This is at odds with the common understanding of ion-molecule reactions, but our model provides a rationale for such apparent incongruity. The strong anisotropic interactions in the $\mathrm{He}^{+}-\mathrm{DME} / \mathrm{MF}$ systems drive the collision complexes into the most attractive configurations, which happen to be the least efficient for charge exchange due to unfavourable molecular overlap.

\subsection{Branching ratios}

The difference between our measured BRs (Cernuto et al. 2017 2018) and those reported in the KIDA and UDfA databases is striking, as shown in the Tables 1 and 2. The uncertainties on our experimental BRs derive from error propagation in the cross section measurements.

In the DME case, while the KIDA database assumes a BR of $50.0 \%$ for the production of $\mathrm{CH}_{3}^{+}$and $\mathrm{OCH}_{3}^{+}$, our results suggest that the most abundant product is $\mathrm{HCO}^{+}(\mathrm{BR}=53.6 \%)$ and that $\mathrm{CH}_{3}{ }^{+}$and $\mathrm{OCH}_{3}^{+}$have branching ratios of $38.5 \%$ and $0.4 \%$, respectively. Results for MF show an even greater disagreement, with $\mathrm{HCO}^{+}$being the most abundant product according to our experiments (with a $\mathrm{BR}=83.2 \%$ ), while both KIDA and UDfA assume the exclusive formation of $\mathrm{HOCO}^{+}$.

\section{Revised values for the reactions of $\mathrm{HCO}^{+}$and $\mathrm{H}_{3}{ }^{+}$with DME and MF}

For completeness, here we review the rate coefficients for the other two ions that dominate the destruction pathways for iCOMs (see Introduction).

\section{1. $\mathrm{HCO}^{+}+\mathrm{DME} / \mathrm{MF}$}

An experimental determination of the rate constants for the proton transfer reaction of $\mathrm{HCO}^{+}$with $\mathrm{DME}$ and $\mathrm{MF}$ at $298 \mathrm{~K}$ exists (Tanner et al. 1979) and gives the following results: $k=$ $(2.1 \pm 0.5) \times 10^{-9} \mathrm{~cm}^{3} \mathrm{~s}^{-1}$ for DME and $(2.9 \pm 0.7) \times 10^{-9} \mathrm{~cm}^{3} \mathrm{~s}^{-1}$ for MF. In both cases, the proton transfer is non dissociative. However, the KIDA database recommends to use the value from the modified Arrhenius equation (see Eq. (1)) with the following parameters: $\alpha=1.2 \times 10^{-9}$ for DME and $1.55 \times 10^{-9}$ for MF, with $\beta=-0.50$ and $\gamma=0.00$ for both. At $298 \mathrm{~K}$ the resulting rate
Table 2. Product branching ratios (BRs) for the reaction $\mathrm{He}^{+}+\mathrm{MF}$.

\begin{tabular}{lccccc}
\hline \hline \multirow{2}{*}{ Product } & $m / z$ & \multicolumn{3}{c}{ BRs (\%) } & \\
\cline { 3 - 4 } \cline { 3 - 4 } & & Exp. values & KIDA & UDfA \\
\hline $\mathrm{CH}_{2}{ }^{+\bullet}$ & 14 & $3.6 \pm 0.3$ & 0 & 0 \\
$\mathrm{CH}_{3}{ }^{+}$ & 15 & $7.3 \pm 0.3$ & 0 & 0 \\
$\mathrm{HCO}^{+}$ & 29 & $83.2 \pm 2.0$ & 0 & 0 \\
$\mathrm{OCH}_{3}{ }^{+}$ & 31 & $4.2 \pm 0.8$ & 0 & 0 \\
$\mathrm{CO}_{2}^{+}$ & 44 & $1.3 \pm 0.1$ & 0 & 0 \\
$\mathrm{HC}(\mathrm{O}) \mathrm{O}^{+}$ & 45 & $0.4 \pm 0.1$ & 100 & 100 \\
\hline
\end{tabular}

Notes. Values are obtained, at a collision energy in the CM frame of $\sim 0.9 \mathrm{eV}$, by averaging results measured at pressures of MF between $1.8 \times 10^{-7}$ and $1.4 \times 10^{-6}$ mbar. BRs show no change with varying collision energy over the explored range.

constants are $k=1.2 \times 10^{-9} \mathrm{~cm}^{3} \mathrm{~s}^{-1}$ for DME and $1.56 \times$ $10^{-9} \mathrm{~cm}^{3} \mathrm{~s}^{-1}$ for MF, i.e. smaller than the measured values by about a factor of two.

Conversely, the UDfA database suggests using the experimental data of Tanner et al. (1979) at $298 \mathrm{~K}$ and rescales them with temperature according to Eq. (1), giving $k_{300}=2.1 \times$ $10^{-9} \mathrm{~cm}^{3} \mathrm{~s}^{-1}$ (for DME) and $2.9 \times 10^{-9} \mathrm{~cm}^{3} \mathrm{~s}^{-1}$ (for MF), with $\beta=-0.50$ for both. At $10 \mathrm{~K}$ the resulting rate constants are $1.15 \times 10^{-8} \mathrm{~cm}^{3} \mathrm{~s}^{-1}$ for DME and $1.58 \times 10^{-8} \mathrm{~cm}^{3} \mathrm{~s}^{-1}$ for MF. For both systems accuracy in the rate constants is given within $\pm 25 \%$. In the following section, we adopt the UDfA recommendations.

\section{2. $\mathrm{H}_{3}^{+}+\mathrm{DME} / \mathrm{MF}$}

Dimethyl ether. Rate coefficients and BRs for the reaction of $\mathrm{H}_{3}{ }^{+}$ with DME have been measured using a flowing afterglow technique at $300 \mathrm{~K}$ (Lee et al. 1992) and the results are as follows: the overall rate coefficient is $4.7 \times 10^{-9} \mathrm{~cm}^{3} \mathrm{~s}^{-1}$ and the proton transfer is highly dissociative giving the following fragments, with $\%$ BRs in brackets: $\mathrm{CH}_{3}{ }^{+}(29 \%), \mathrm{CH}_{5}{ }^{+}(8 \%), \mathrm{HCO}^{+} / \mathrm{C}_{2} \mathrm{H}_{5}{ }^{+}(10 \%)$, $\mathrm{CH}_{3} \mathrm{O}^{+}(26 \%), \mathrm{C}_{2} \mathrm{H}_{5} \mathrm{O}^{+}$(15\%), $\mathrm{CH}_{3} \mathrm{OHCH}_{3}{ }^{+}(12 \%)$.

The KIDA data is at odds with these findings, as it exclusively quotes protonated DME $\left(\mathrm{CH}_{3} \mathrm{OHCH}_{3}{ }^{+}\right)$as a product with $k=3.01 \times 10^{-9} \mathrm{~cm}^{3} \mathrm{~s}^{-1}$ at $298 \mathrm{~K}$ (from the modified Arrhenius Eq. (1) with parameters $\left.\alpha=3.00 \times 10^{-9}, \beta=-0.50, \gamma=0.00\right)$. The UDfA database similarly gives a rate constant for non dissociative proton transfer in terms of Eq. (1) with $k_{300}=2 \times 10^{-9}$ and $\beta=-0.50$ for $T$ ranging from 10 to $41000 \mathrm{~K}$, with an accuracy of $\pm 50 \%$ in the rate constant.

Hereafter, we use the total $\alpha=4.70 \times 10^{-9}$, BRs as given by Lee et al. (1992), $\beta=-0.50$ and $\gamma=0.00$ over the $10-300 \mathrm{~K}$ temperature range. In order to avoid introducing new trace ions into the network, we will consider only the two most abundant channels, namely those giving $\mathrm{CH}_{3}{ }^{+}$and $\mathrm{CH}_{3} \mathrm{O}^{+}$as products, and the channel relative to the undissociative proton-trasfer leading to $\mathrm{CH}_{3} \mathrm{OHCH}_{3}{ }^{+}$plus $\mathrm{H}_{2}$ (already included in the models with a different rate coefficient).

Methyl formate. In the MF case no measurements for the rate coefficients have been performed. However, Lawson et al. (2012), while attempting to measure the rate constants for dissociative electron-ion recombination of protonated $\mathrm{MF}$, observed that significant fragmentation occurred when attempting to induce proton transfer from $\mathrm{H}_{3}{ }^{+}$to MF. In addition, according to a chemical ionisation mass spectrometric experimental study (Herman \& Harrison 1981), the outcome of the protonation reaction of $\mathrm{MF}$ by $\mathrm{H}_{3}{ }^{+}$is mostly dissociative and the observed 
relative abundances of ionised fragments are: $15.5 \%$ for $\mathrm{H}_{5} \mathrm{C}_{2} \mathrm{O}_{2}{ }^{+}, 74 \%$ for $\mathrm{CH}_{3} \mathrm{OH}_{2}{ }^{+}, 5.9 \%$ for $\mathrm{CH}_{2} \mathrm{OH}^{+}$and $4.4 \%$ for $\mathrm{CH}_{3}{ }^{+}$.

Such experimental evidence is at odds with the prescriptions from KIDA, where the reaction is assumed to be completely non dissociative with $k=4.06 \times 10^{-9} \mathrm{~cm}^{3} \mathrm{~s}^{-1}$ at $298 \mathrm{~K}$ (from the modified Arrhenius Eq. (1) with parameters $\alpha=$ $4.05 \times 10^{-9}, \beta=-0.50$ and $\left.\gamma=0.00\right)$. The value reported in the UDfA database is Eq. (1) with $k_{300}=3 \times 10^{-9}$ and $\beta=-0.50$ (for a $T$ range $10-41000 \mathrm{~K})$.

In the following, we adopt the KIDA value for the total rate constant (i.e. $\alpha=4.05 \times 10^{-9}, \beta=-0.50$ and $\gamma=0.00$ ), while for the BRs we will use the values found by Herman \& Harrison (1981). As before, to avoid introducing new trace ions in the network, we will consider only the two most abundant channels, namely those producing $\mathrm{CH}_{3} \mathrm{OH}_{2}{ }^{+}$and protonated MF.

\section{Astrophysical implications}

\subsection{New values to adopt in the astrochemical networks}

From the discussion in Sect. 3, it is clear that the new rate coefficients, based on the experimental results by Cernuto et al. (2017, 2018), differ from the those reported in both KIDA and UDfA databases in terms of their absolute values, their temperature dependence (which is positive rather than negative) as well as in the product BRs.

An interpolation of the new rate coefficients (and extrapolated values at very low temperatures) gives the following parameters for the global destruction rate coefficients of DME and MF through reaction with $\mathrm{He}^{+}: \alpha=1.4 \times 10^{-9}, \beta=+0.295$, $\gamma=0.00$ for DME and $\alpha=1.4 \times 10^{-9}, \beta=+0.241, \gamma=0.00$ for MF. These values lead to rate coefficients much smaller than those adopted in astrochemical databases, especially at the low temperatures (below $50 \mathrm{~K}$ ) of relevance in the ISM. The total rate coefficients must then be combined with the experimental BRs reported in Sect. 3 to give the rates for the individual channels.

The product BRs were seen to be constant over a wide range of temperatures, so that we can reliably assume that they are also valid at the low temperatures of relevance to the ISM. Given that the channels with the largest yields, accounting globally for more than $80 \%$ of the reactions, are those leading to $\mathrm{HCO}^{+}$and $\mathrm{CH}_{3}{ }^{+}$ for $\mathrm{DME}$ and the one leading to $\mathrm{HCO}^{+}$for $\mathrm{MF}$, we recommend that only these selected channels are included when updating the astrochemical databases (the small yields of the other channels make the effects of their inclusion in astrochemical models negligible for all purposes).

Finally, we have to note that the present experimental results allow us to infer the chemical nature of the formed ionic products, but additional combinations of neutral co-products are indeed possible. In particular:

1. For $D M E$, as co-fragments of $\mathrm{HCO}^{+}$, we can have either $\mathrm{CH}_{3}+\mathrm{H}_{2}$, or $\mathrm{CH}_{3}+2 \mathrm{H}$, while for $\mathrm{CH}_{3}{ }^{+}$we can have either $\mathrm{CH}_{3} \mathrm{O}$ or $\mathrm{H}_{2} \mathrm{CO}+\mathrm{H}$;

2. For $\mathrm{MF}$, as co-fragments of $\mathrm{HCO}^{+}$, we can have either $\mathrm{CH}_{3} \mathrm{O}, \mathrm{CH}_{2} \mathrm{OH}$, or $\mathrm{HCO}+\mathrm{H}_{2}$.

In the absence of any experimental information, we considered all of these combinations to be equally probable. We adopted a similar procedure to estimate the products of the DME fragmentation from the reaction with $\mathrm{H}_{3}{ }^{+}$(see Sect. 4). Table 3 summarises the new values that we recommend to be used in the astrochemical networks.
Table 3. Summary of products and rates of the reactions of $\mathrm{He}^{+}, \mathrm{HCO}^{+}$, and $\mathrm{H}_{3}{ }^{+}$with DME and MF, respectively.

\begin{tabular}{|c|c|c|c|}
\hline Reactants & Products & $\alpha\left(\times 10^{-10}\right)$ & $\beta$ \\
\hline $\mathrm{CH}_{3} \mathrm{OCH}_{3}+\mathrm{He}^{+}$ & $\mathrm{He}+\mathrm{CH}_{3}{ }^{+}+\mathrm{CH}_{3} \mathrm{O}$ & 2.9 & 0.295 \\
\hline $\mathrm{CH}_{3} \mathrm{OCH}_{3}+\mathrm{He}^{+}$ & $\mathrm{He}+\mathrm{CH}_{3}{ }^{+}+\mathrm{H}_{2} \mathrm{CO}+\mathrm{H}$ & 2.9 & 0.295 \\
\hline $\mathrm{CH}_{3} \mathrm{OCH}_{3}+\mathrm{He}^{+}$ & $\mathrm{He}+\mathrm{HCO}^{+}+\mathrm{CH}_{3}+\mathrm{H}_{2}$ & 4.0 & 0.295 \\
\hline $\mathrm{CH}_{3} \mathrm{OCH}_{3}+\mathrm{He}^{+}$ & $\mathrm{He}+\mathrm{HCO}^{+}+\mathrm{CH}_{3}+2 \mathrm{H}$ & 4.0 & 0.295 \\
\hline $\mathrm{HCOOCH}_{3}+\mathrm{He}^{+}$ & $\mathrm{He}+\mathrm{HCO}^{+}+\mathrm{CH}_{3} \mathrm{O}$ & 4.6 & 0.241 \\
\hline $\mathrm{HCOOCH}_{3}+\mathrm{He}^{+}$ & $\mathrm{He}+\mathrm{HCO}^{+}+\mathrm{CH}_{2} \mathrm{OH}$ & 4.6 & 0.241 \\
\hline $\mathrm{HCOOCH}_{3}+\mathrm{He}^{+}$ & $\mathrm{He}+\mathrm{HCO}^{+}+\mathrm{HCO}+\mathrm{H}_{2}$ & 4.6 & 0.241 \\
\hline $\mathrm{CH}_{3} \mathrm{OCH}_{3}+\mathrm{HCO}^{+}$ & $\mathrm{CO}+\mathrm{CH}_{3} \mathrm{O}(\mathrm{H}) \mathrm{CH}_{3}{ }^{+}$ & 21 & -0.50 \\
\hline $\mathrm{CH}_{3} \mathrm{OCH}_{3}+\mathrm{H}_{3}^{+}$ & $\mathrm{H}_{2}+\mathrm{CH}_{3}{ }^{+}+\mathrm{CH}_{3} \mathrm{O}+\mathrm{H}$ & 10 & -0.50 \\
\hline $\mathrm{CH}_{3} \mathrm{OCH}_{3}+\mathrm{H}_{3}^{+}$ & $\mathrm{H}_{2}+\mathrm{CH}_{3}{ }^{+}+\mathrm{H}_{2} \mathrm{CO}+\mathrm{H}_{2}$ & 1 & -0.50 \\
\hline $\mathrm{CH}_{3} \mathrm{OCH}_{3}+\mathrm{H}_{3}^{+}$ & $\mathrm{H}_{2}+\mathrm{CH}_{3} \mathrm{O}^{+}+\mathrm{CH}_{4}$ & 18 & -0.50 \\
\hline $\mathrm{CH}_{3} \mathrm{OCH}_{3}+\mathrm{H}_{3}^{+}$ & $\mathrm{H}_{2}+\mathrm{CH}_{3} \mathrm{O}(\mathrm{H}) \mathrm{CH}_{3}^{+}$ & 8.4 & -0.50 \\
\hline $\mathrm{HCOOCH}_{3}+\mathrm{HCO}^{+}$ & $\mathrm{CO}+\mathrm{H}_{5} \mathrm{C}_{2} \mathrm{O}_{2}^{+}$ & 29 & -0.50 \\
\hline $\mathrm{HCOOCH}_{3}+\mathrm{H}_{3}{ }^{+}$ & $\mathrm{H}_{2}+\mathrm{CH}_{3} \mathrm{OH}_{2}^{+}+\mathrm{CO}$ & 34 & -0.50 \\
\hline $\mathrm{HCOOCH}_{3}+\mathrm{H}_{3}{ }^{+}$ & $\mathrm{H}_{2}+\mathrm{H}_{5} \mathrm{C}_{2} \mathrm{O}_{2}^{+}$ & 7.0 & -0.50 \\
\hline
\end{tabular}

Notes. The parameter $\gamma$ is assumed to be equal to 0.00 on all reactions.

\subsection{Impact on the abundances}

Our new rate coefficients for the destruction of DME and MF through reaction with $\mathrm{He}^{+}$differ greatly from those given by the astrochemical databases, KIDA and UDfA, especially at the low temperatures of the ISM gas. In particular, with respect to KIDA, our values are smaller by a factor between five and six at $100 \mathrm{~K}$, the temperature of the warm gas in hot corinos and outflow molecular shocks where iCOMs are abundantly detected (e.g. Bottinelli et al. 2004, 2007; Taquet et al. 2015; Jorgensen et al. 2016; Ligterink et al. 2017; Lefloch et al. 2012, 2017, 2018; Codella et al. 2017), and by a factor of 25-28 at $10 \mathrm{~K}$, the temperature of prestellar cores where iCOMs are also detected (Bacmann et al. 2012; Cernicharo et al. 2012; Vastel et al. 2014; Jimenez-Serra et al. 2016)

Additionally, the review of the literature regarding the DME and $\mathrm{MF}$ reactions with $\mathrm{HCO}^{+}$and $\mathrm{H}_{3}{ }^{+}$has also shown important differences between the rate coefficients reported in the KIDA and UDfA databases with respect to the available experimental data, and more accurate experiments and/or theoretical calculations are clearly needed to better understand the products of the reactions and the dependence on temperature.

In this context, we ran a few simulations to quantify the effect of these differences on the predicted abundances. To this end, we used a version revised by us of the Nahoon code, which is publicly available in the KIDA database. Briefly, Nahoon is a pure gas-phase time-dependent code that allows to follow the chemical composition of a gas at a given temperature and density (Wakelam et al. 2012). In our case, we used $T=10 \mathrm{~K}$ and $n_{\mathrm{H}}=2 \times 10^{5} \mathrm{~cm}^{-3}$ to describe a generic prestellar core, and $T=100 \mathrm{~K}$ and $n_{\mathrm{H}}=2 \times 10^{8} \mathrm{~cm}^{-3}$ to describe a generic hot corino. Since the abundance of $\mathrm{He}^{+}$as well as $\mathrm{HCO}^{+}$and $\mathrm{H}_{3}{ }^{+}$depends on the cosmic-ray ionisation rate, $\zeta_{\mathrm{CR}}$, whose value in these regions is known with a large uncertainty (e.g. Padovani et al. 2009; Vaupre et al. 2014), we ran simulations using both a low $\left(10^{-18} \mathrm{~s}^{-1}\right)$ and high $\left(10^{-15} \mathrm{~s}^{-1}\right)$ cosmic ray ionisation rate to model two extreme situation of gas ionisation.

Finally, the initial abundances were chosen as follows. In the prestellar core models, we adopted the abundances from Jenkins (2009), with those of the heavy metals (S, Si, Mg, Fe, Na) being decreased by a factor of 100 and those for the light ones by a factor of ten: $\mathrm{C} / \mathrm{H}=1.7 \times 10^{-5}, \mathrm{~N} / \mathrm{H}=6.2 \times 10^{-6}$, and we changed 
Table 4. Summary of the input values for the simulations that were run to study the impact of the new rate coefficients of the reactions of DME and MF with $\mathrm{He}^{+}, \mathrm{HCO}^{+}$, and $\mathrm{H}_{3}{ }^{+}$on the predicted abundances of DME and MF in prestellar cores, and hot corinos and molecular outflow shocks, respectively.

\begin{tabular}{lcccc}
\hline \hline Model & $\begin{array}{c}\text { Temperature } \\
(\mathrm{K})\end{array}$ & $\begin{array}{c}n_{H} \\
\mathrm{~cm}^{-3}\end{array}$ & $\begin{array}{c}\zeta_{\mathrm{CR}} \\
10^{-17} \mathrm{~s}^{-1}\end{array}$ & Initial abundances \\
\hline 1 & 10 & $2 \times 10^{5}$ & 0.1 & Modified Jenkins (2009); $\mathrm{O} / \mathrm{H}=2.6 \times 10^{-5}$ \\
2 & 10 & $2 \times 10^{5}$ & 0.1 & Modified Jenkins (2009); $\mathrm{O} / \mathrm{H}=1.8 \times 10^{-5}$ \\
3 & 10 & $2 \times 10^{5}$ & 100 & Modified Jenkins (2009); O/H=2.6 $\mathrm{H} 10^{-5}$ \\
4 & 10 & $2 \times 10^{5}$ & 100 & Modified Jenkins (2009); O/H =1.8 $\times 10^{-5}$ \\
5 & 100 & $2 \times 10^{8}$ & 0.1 & Codella et al. (2017) \\
6 & 100 & $2 \times 10^{8}$ & 100 & Codella et al. (2017) \\
\hline
\end{tabular}

Notes. For the details on the initial abundances, see text.

$\mathrm{O} / \mathrm{H}$ ratio from $2.6 \times 10^{-5}$ to $1.8 \times 10^{-5}$ to simulate the segregation of oxygen on the water ices. In the hot corino and molecular outflow models, we used the initial abundances described in Codella et al. (2017). The parameters used in the models that we run are summarised in Table 4.

We first ran simulations with our old chemical network, which is based on the KIDA 2014 network $^{5}$, upgraded with the reactions described in Balucani et al. (2015), Skouteris et al. (2018) and Rosi et al. (2018). We then changed the reactions with $\mathrm{He}^{+}, \mathrm{HCO}^{+}$and $\mathrm{H}_{3}{ }^{+}$according to Table 3 and studied the changes in the predicted abundances. We note that our goal here is not to carry out a full study of the impact of the new reaction products and rate coefficients, but only to estimate whether this would dramatically change the model predictions in "standard" situations where DME and MF have been detected.

The effect is indeed dependent on the parameters that we have explored, details of which are given in Table 4. In cold gas and conditions similar to the prestellar cores the abundances of DME and MF can be different by up to $40 \%$, with the difference larger when the oxygen abundance is greater (models 1 and 3 ). This effect does seems to depend little on the cosmic-ray ionisation rate used. This is also the case for the models simulating the hot corino gas, where the difference in the abundances are smaller (about $\leq 10 \%$ ), as expected based on the difference in the rate coefficients at $100 \mathrm{~K}$. This is because, in all these models, the DME and MF destruction is dominated by $\mathrm{H}_{3}{ }^{+}$, with the $\mathrm{HCO}^{+}$ and $\mathrm{He}^{+}$pathways proceeding at a slower rate by about a factor of ten. We emphasise that, although our pure gas-phase model predicts low $\left(\leq 10^{-14}\right)$ abundances for DME and MF, the effect may be larger if a full model, such as that used in Balucani et al. (2015), is employed, though this is beyond the scope of this work.

\section{Conclusions}

We have reported a new study of the branching ratios, products and rate coefficients for the reactions of DME and MF with $\mathrm{He}^{+}$ and we have reviewed the destruction routes of DME and MF by the other two abundant ions in molecular gas, namely $\mathrm{H}_{3}{ }^{+}$and $\mathrm{HCO}^{+}$.

The main conclusions of this work are summarized below. Firstly, we have computed branching ratios, products and rate coefficients of $\mathrm{DME}$ and $\mathrm{MF}+\mathrm{He}^{+}$. These calculations are based on the recent measurements and theoretical modelling of the cross-sections performed by Cernuto et al. (2017, 2018). The newly determined values substantially differ from those

\footnotetext{
5 http://kida.obs.u-bordeaux1.fr
}

reported in the commonly used astrochemical databases KIDA and UDfA.

Secondly, the dependence of the rate coefficients on the temperature is positive and not negative, as assumed in the KIDA and UDfA databases. As a consequence, the difference in the rate coefficients is larger at low temperatures: at $10 \mathrm{~K}$ the values differ by more than a factor of 20.

Thirdly, the review of the DME/MF $+\mathrm{H}_{3}{ }^{+} / \mathrm{HCO}^{+}$reactions show that BRs, products and rate coefficients of these reactions are poorly understood. Nonetheless, the data available in the literature differ from that used in the KIDA and UDfA databases. We provide a table of the parameters to include in the astrochemical networks that we recommend for the six reactions of DME and $\mathrm{MF}$ with $\mathrm{He}^{+}, \mathrm{H}_{3}{ }^{+}$and $\mathrm{HCO}^{+}$.

A very general modelling of cold $(10 \mathrm{~K})$ and warm $(100 \mathrm{~K})$ gas simulating the conditions where DME and MF are detected, namely prestellar cores, hot cores and corinos, shows that the new rates have an impact on the predicted abundances, of up to $\sim 40 \%$. However, different conditions might lead to even larger differences.

In conclusion, we emphasise the importance of reliable information on the destruction pathways of iCOMs by the most abundant ions in molecular gas: $\mathrm{H}_{3}{ }^{+}, \mathrm{HCO}^{+}$and $\mathrm{He}^{+}$. This can be obtained with experimental works, as in the case reported here (Cernuto et al. 2017, 2018) coupled with theoretical modelling, or by ab initio quantum chemistry calculations.

A more general conclusion is that attention should be paid when using estimated rate coefficients for unknown processes or when extrapolating room temperature rate coefficients at very low temperatures. In the case of the reactions between $\mathrm{He}^{+}$and DME/MF the results obtained with a very detailed treatment of the entrance channel challenge the common notion that ionmolecule reaction rate coefficients increase with decreasing $T$ (or remain constant as in the Langevin model). In addition, we note that product branching ratios are difficult to guess in the absence of experimental or theoretical results. We recommend that the physical chemistry community dedicate as much effort as possible to characterise all the reactions which play a significant role in astrochemical models.

Acknowledgements. This work was supported by the Italian Ministero dell'Istruzione, Universitá e Ricerca through the grant Progetti Premiali 2012 iALMA (CUP C52I13000140001). This project has received funding from the European Research Council (ERC) under the European Union's Horizon 2020 research and innovation programme, for the Project "The Dawn of Organic Chemistry" (DOC), grant agreement No 741002. The support of the Department of Physics of the University of Trento is gratefully acknowledged. N.B. and F.P. acknowledge the financial support from Italian MIUR "PRIN 2015", project 
"STARS in the CAOS (Simulation Tools for Astrochemical Reactivity and Spectroscopy in the Cyberinfrastructure for Astrochemical Organic Species)", grant number 2015F59J3R. We are grateful to Mr. Vincent Richardson, from the Dept Physics - University of Trento, for his precious help with English Language editing and revision.

\section{References}

Bacmann, A., Taquet, V., Faure, A., Kahane, C., \& Ceccarelli, C. 2012, A\&A 541, L12

Balucani, N., Ceccarelli, C., \& Taquet, V. 2015, MNRAS, 449, L16

Blake, G. A., Sutton, E. C., Masson, C. R., \& Phillips, T. G. 1987, ApJ, 315, 621

Bottinelli, S., Ceccarelli, C., Lefloch, B., et al. 2004, ApJ, 615, 354

Bottinelli, S., Ceccarelli, C., Williams, J. P., \& Lefloch, B. 2007, A\&A, 463, 601

Ceccarelli, C., Caselli, P., Fontani, F., et al. 2017, AJ, 850, 176

Cernicharo, J., Marcelino, N., Roueff, E., et al. 2012, ApJ, 759, L43

Cernuto, A., Tosi, P., Martini, L. M., Pirani, F., \& Ascenzi, D. 2017, Phys. Chem. Chem. Phys., 19, 19554

Cernuto, A., Ascenzi, D., Pirani, F., Martini, L. M., \& Tosi, P. 2018, ChemPhysChem, 19, 51

Chesnavich, W. J., Su, T., \& Bowers, M. T. 1980, J. Chem. Phys., 72, 2641

Codella, C., Ceccarelli, C., Caselli, P., et al. 2017, A\&A, 605, A110

Falcinelli, S., Rosi, M., Cavalli, S., Pirani, F., \& Vecchiocattivi, F. 2016, Chem. Eur. J., 22, 12518

Garrod, R. T., \& Herbst, E. 2006, A\&A, 457, 927

Geppert, W. D., Hamberg, M., Thomas, R. D., et al. 2006, Faraday Discuss., 133, 177

Gislason, E. A., \& Sachs, J. G. 1975, J. Chem. Phys., 62, 2678

Gislason, E. A., Parlant, G., \& Sizun, M. 2007, The Semiclassical TimeDependent Approach to Charge-Transfer Processes (John Wiley and Sons, Inc.), 321

Hamberg, M., Österdahl, F., Thomas, R. D., et al. 2010, A\&A, 514, A83

Herbst, E. 2009, OSU2009, http://faculty.virginia.edu/ericherb/ research_files/osu_01_2009

Herbst, E., \& van Dishoeck, E. F. 2009, Annu. Rev. Astron. Astrophys., 47, 427
Herman, J. A., \& Harrison, A. G. 1981, Can. J. Chem., 59, 2133

Jaber, A. A., Ceccarelli, C., Kahane, C., \& Caux, E. 2014, ApJ, 791, 29 Jenkins, E. B. 2009, ApJ, 700, 1299

Jimenez-Serra, I., Vasyunin, A. I., Caselli, P., et al. 2016, ApJ, 830, L6

Jorgensen, J. K., van der Wiel, M. H. D., Coutens, A., et al. 2016, A\&A, 595, A 117

Lawson, P. A., Osborne, D. S., \& Adams, N. G. 2012, J. Phys. Chem. A, 116, 2880

Lee, H. S., Drucker, M., \& Adams, N. G. 1992, Int. J. Mass Spectrom. Ion Processes, 117, 101

Lefloch, B., Cabrit, S., Busquet, G., et al. 2012, ApJ, 757, L25

Lefloch, B., Ceccarelli, C., Codella, C., et al. 2017, MNRAS, 469, L73

Lefloch, B., Bachiller, R., Ceccarelli, C., et al. 2018, MNRAS, 477, 4792

Ligterink, N. F. W., Coutens, A., Kofman, V., et al. 2017, MNRAS, 469, 2219

Maergoiz, A., Nikitin, E., \& Troe, J. 2009, Int. J. Mass Spectrom., 280, 42

McElroy, D., Walsh, C., Markwick, A. J., et al. 2013, A\&A, 550, A36

Padovani, M., Galli, D., \& Glassgold, A. E. 2009, A\&A, 501, 619

Rosi, M., Mancini, L., Skouteris, D., et al. 2018, Chem. Phys. Lett., 695, 87

Ruaud, M., Loison, J. C., Hickson, K. M., et al. 2015, MNRAS, 447, 4004

Rubin, R. H., Swenson, Jr., G. W., Benson, R. C., Tigelaar, H. L., \& Flygare, W. H. 1971, ApJ, 169, L39

Shingledecker, C. N., Tennis, J., Le Gal, R., \& Herbst, E. 2018, AJ, 861, A20

Skouteris, D., Vazart, F., Ceccarelli, C., et al. 2017, MNRAS, 468, L1

Skouteris, D., Balucani, N., Ceccarelli, C., et al. 2018, AJ, 854, 135

Skouteris, D., Balucani, N., Ceccarelli, C., et al. 2019, MNRAS, 482, 3567

Su, T., \& Chesnavich, W. J. 1982, J. Chem. Phys., 76, 5183

Tanner, S. D., Mackay, G. I., Hopkinson, A. C., \& Bohme, A. C. 1979, Int. J. Mass Spectrom. Ion Processes, 29, 153

Taquet, V., Lopez-Sepulcre, A., Ceccarelli, C., et al. 2015, AJ, 804, A81

Troe, J. 1996, J. Chem. Phys., 105, 6249

Vastel, C., Ceccarelli, C., Lefloch, B., \& Bachiller, R. 2014, ApJ, 795, L2

Vasyunin, A., \& Herbst, E. 2013, AJ, 769, 34

Vasyunin, A. I., Caselli, P., Dulieu, F., \& Jimenez-Serra, I. 2017, AJ, 842, A33

Vaupre, S., Hily-Blant, P., Ceccarelli, C., et al. 2014, A\&A, 568, A50

Wakelam, V., Smith, I. W. M., Herbst, E., et al. 2010, Space Sci. Rev., 156, 13

Wakelam, V., Herbst, E., Loison, J., et al. 2012, ApJS, 199, 21 